\title{
Knot concordance and Heegaard Floer homology invariants in branched covers
}

\author{
J ELISENDA GRIGSBY \\ DANIEL RUBERMAN \\ SAŠO STRLE
}

\begin{abstract}
By studying the Heegaard Floer homology of the preimage of a knot $K \subset S^{3}$ inside its double branched cover, we develop simple obstructions to $K$ having finite order in the classical smooth concordance group. As an application, we prove that all 2-bridge knots of crossing number at most 12 for which the smooth concordance order was previously unknown have infinite smooth concordance order.
\end{abstract}

57R58, 57M25; 57M12, 57M27

\section{Introduction}

The introduction of a number of invariants has revitalized the study of classical knot concordance in recent years. In this paper, we combine two of the most powerful of these new invariants, the correction term $d$ defined by Ozsváth-Szabó in [27] and the smooth concordance invariant $\tau$ defined by Ozsváth-Szabó in [23] and Rasmussen in [28], with older techniques due to Casson and Gordon [1], to give new obstructions to a knot being smoothly slice. Our main results imply that many knots in the knot table (cf Knotinfo [3] or Cha-Livingston [2]) in fact have infinite order in the smooth concordance group. These are knots whose order is not detected by classical methods, nor by recently introduced concordance invariants such as the Ozsváth-Szabó $\tau$-invariant [23], Rasmussen $s$-invariant [29;30], or Manolescu-Owens $\delta$-invariant [18]. Our results support a conjecture of Gordon [11, Problem 1.32] that there are no torsion elements in the smooth concordance group with order greater than 2 .

The methods in this paper apply exclusively in the smooth category. It is conceivable that the knots we consider might be shown to be of infinite order in the topological knot concordance group, by clever applications of the Casson-Gordon invariants, or by the sophisticated methods deriving from the work of Cochran-Orr-Teichner [4].

Our approach relies on the study of the $d$ and $\tau$-invariants associated to the preimage of a knot $K \subset S^{3}$ in a cyclic branched cover (cf Grigsby [8; 7]). Let $K \subset S^{3}$, and 
$Y \rightarrow S^{3}$ be the $n$-fold cyclic branched cover of $K$ with $n=q^{r}$ where $q$ is a prime. It is well-known that $Y$ is a rational homology sphere $\left(\mathbb{Q} H S^{3}\right)$, and that if $K$ is slice, then the corresponding branched cover $W$ of the 4-ball branched along the slice disk $\Delta$ is a rational homology ball $\left(\mathbb{Q} H^{4}\right)$. As observed by Ozsváth and Szabó [22] and elaborated in Owens-Strle [21], Manolescu-Owens [18] and Jabuka-Naik [9], this implies that many of the Ozsváth-Szabó $d$-invariants must vanish.

However, there is more information in the branched cover, as the branch set $\widetilde{K}$ is the boundary of the lift $\widetilde{\Delta}$ of $\Delta$ to $W$. Now the knot $\widetilde{K} \subset Y$ has a collection $\left\{\tau_{\mathfrak{s}}(Y, \widetilde{K})\right\}$ of $\tau$-invariants, one for each $\mathfrak{s} \in \operatorname{Spin}^{c}(Y)$. In this situation, we prove the following vanishing theorem for those $\tau_{\mathfrak{s}}$ associated to $\operatorname{spin}^{c}$ structures extending over $W$.

Theorem 1.1 Let $K$ be a knot in $S^{3}$, and $Y$ the $q^{r}$-fold cover of $S^{3}$ branched along $K$. Denote by $\widetilde{K}$ the preimage of $K$ in $Y$. If $K$ is slice, then there exists a subgroup $G<H^{2}(Y ; \mathbb{Z})$ with $|G|^{2}=\left|H^{2}(Y ; \mathbb{Z})\right|$ such that $d_{\mathfrak{s}}(Y)=0$ and $\tau_{\mathfrak{s}}(\widetilde{K})=0$ for all $\mathfrak{s} \in \mathfrak{s}_{0}+G$, where $\mathfrak{s}_{0}$ is the spin structure on $Y$ described in Lemma 2.1.

As in many variations on the Casson-Gordon theme, it takes some work to extract computable obstructions from this theorem. One important issue is that one does not know a priori which $\operatorname{spin}^{c}$ structures on $Y$ extend over $W$. This problem gets worse when one studies the order of $K$ in the knot concordance group, because the number of $\operatorname{spin}^{c}$ structures that need to be examined can be very large. (Compare the discussion in Jabuka-Naik [9, Section 5].) In addition, although there have been considerable advances in the computability of Heegaard Floer homology invariants in recent months (cf Manolescu-Ozsváth-Sarkar [19] and Sarkar-Wang [31]), much remains out of reach.

Even within the limited scope allowed by the current technology, however, one can deduce a considerable amount of information about knot concordance. In particular, concentrating on the case $q^{r}=2$, we define in Section 4 , for each prime $p$, two invariants, $\mathcal{T}_{p}(K) \in \mathbb{Z}$ and $\mathcal{D}_{p}(K) \in \mathbb{Q}$ that vanish on knots $K$ with finite smooth concordance order. These obstructions are particularly simple whenever $H^{2}(Y, \mathbb{Z})$ is cyclic for $Y$ the double-branched cover of $K$ (as occurs, for example, for all 2-bridge knots).

Theorem 1.2 Let $K \subset S^{3}$ be a knot and $p \in \mathbb{Z}_{+}$prime or 1 . If there exists a positive $n \in \mathbb{Z}$ such that $\#_{n} K$ is smoothly slice, then $\mathcal{T}_{p}(K)=\mathcal{D}_{p}(K)=0$.

Computation of these new invariants using the algorithm described in Grigsby [7] to compute $\tau$-invariants and the inductive formula in Ozsváth-Szabó [22] for $d$-invariants allows us to determine the smooth concordance order of all 2-bridge knots of 12 or fewer crossings for which the smooth concordance order was previously unknown. 
Theorem 1.3 All 2-bridge knots ${ }^{1}$ of 12 or fewer crossings have smooth concordance order 1,2 or $\infty$.

It is also worthwhile to note that $\mathcal{D}_{p}(K)$ can be computed in a straightforward manner for knots with alternating diagrams and has been quite successful in obstructing finite concordance order in many cases where other tests fail. In particular, Adam Levine [13] has recently used $\mathcal{D}_{p}(K)$ to show that forty-six of the sixty-seven knots whose concordance orders were previously unknown have infinite concordance order.

After this paper was submitted, Paolo Lisca $[14 ; 15]$ determined the smooth concordance order of all 2-bridge knots, using Donaldson's theorem [6] on the intersection form of closed smooth 4-manifolds. His result implies, among other things, that the conclusion of Theorem 1.3 holds for all 2-bridge knots, without restriction on the number of crossings.

We are confident that as computational techniques improve, we will be able to gather similar results for a wider class of knots. We remark that there are many methods (for example Jiang [10], Livingston-Naik [16; 17] and Cochran-Orr-Teichner [5]) for showing that a knot with finite order in the algebraic concordance group has infinite smooth or topological concordance order. There is some overlap between results deduced by these methods and results from our paper.

The paper is organized as follows. In Section 2, we discuss $\operatorname{spin}^{c}$ structures on branched covers of $B^{4}$. In Section 3, we give a proof of Theorem 1.1, along with some relevant Heegaard Floer homology background. In Section 4 we define $\mathcal{T}_{p}$ and $\mathcal{D}_{p}$, prove that they provide obstructions to finite smooth concordance order, and discuss some related, more general obstructions. In Section 5, we apply our results to all 2-bridge knots of 12 or fewer crossings. We also show that the twist knots, with the exception of the figure- 8 and stevedore's knot, have infinite order in the knot concordance group. We conclude, in Section 6, with details about the $\tau$ computations.

We thank Chuck Livingston for his help in the use of Knotinfo [3] and Jiajun Wang for some useful suggestions regarding the Floer homology computation algorithm. The first author would also like to thank Peter Ozsváth for some enlightening discussions during the course of this work. The first author was partially supported by an NSF Postdoctoral Fellowship. The second author was partially supported by NSF Grant 0505605 . The third author was supported in part by the Slovenian Research Agency program No. P1-0292-0101-04 and project No. J1-6128-0101-04. Visits of the second and third authors were supported by a Slovenian-USA Research Project BI-US/06-07/003 and by the NSF.

\footnotetext{
${ }^{1} K_{p, q}$ denotes the 2 -bridge knot whose double-branched cover is the lens space $-L(p, q)$.
} 


\section{Lifting a spin structure}

Theorem 1.1 asserts the vanishing of the $\tau$-invariants on those $\operatorname{spin}^{c}$ structures on the $q^{r}$-fold covers of $S^{3}$ branched along a knot $K$ that extend over the corresponding branched cover of the 4 -ball. The cohomology group $H^{2}(Y ; \mathbb{Z})$ acts freely and transitively on the set of $\operatorname{spin}^{c}$ structures on a manifold $Y$, and so a choice of one $\operatorname{spin}^{c}$ structure gives a bijection between that set and $H^{2}(Y ; \mathbb{Z})$. We will show that there is a canonical $\operatorname{spin}^{c}$ structure, in fact a spin structure, on $Y$ that extends over the branched cover of the 4 -ball. This is the spin structure $\mathfrak{s}_{0}$ referred to in Theorem 1.1.

Lemma 2.1 Let $p:(W, \widetilde{F}) \rightarrow\left(B^{4}, F\right)$ be an $n$-fold cyclic branched cover with branch set a connected surface $F$. Then there is a unique spin structure $\mathfrak{s}_{0}$ on $W$ characterized as follows: if $n$ is odd, the restriction of $\mathfrak{s}_{0}$ to $W-v(\widetilde{F})$ is the pullback $\widetilde{\mathfrak{s}}$ of the spin structure on $B^{4}-v(F)$ that extends over $B^{4}$, whereas if $n$ is even, the restriction of $\mathfrak{s}_{0}$ to $W-v(\widetilde{F})$ is $\widetilde{\mathfrak{s}}$ twisted by the element of $H^{1}\left(W-v(\widetilde{F}) ; \mathbb{Z}_{2}\right)$ supported on the linking circle of $\widetilde{F}$.

Proof Let $\mathfrak{s}$ be the spin structure on $B^{4}-v(F)$ that extends over $B^{4}$ and let $\widetilde{\mathfrak{s}}$ be its pullback to $W-v(\widetilde{F})$. Then $\widetilde{\mathfrak{s}}$ extends over $W$ iff its restriction to the circle bundle $S(\widetilde{F})$ extends over the disk bundle $v(\widetilde{F})$. This happens precisely when the restriction to the fibre $S^{1}$ extends over the disk $B^{2}$. Recall that a spin structure on an oriented manifold $M$ corresponds to a cohomology class in $H^{1}\left(F(M) ; \mathbb{Z}_{2}\right)$ where $F(M)$ is the oriented frame bundle. Clearly $F\left(S^{1}\right)=S^{1}$, and one can check that the spin structure on $S^{1}$ that extends over $B^{2}$ corresponds to the nontrivial element of $H^{1}\left(S^{1} ; \mathbb{Z}_{2}\right)$. Since the covering map is of order $n, \widetilde{\mathfrak{s}}$ "inherits" this property from $\mathfrak{s}$ if $n$ is odd. If $n$ is even, $\widetilde{\mathfrak{s}}$ does not extend over the disks. However, since the class of the fibre is of infinite order in $H_{1}(W-v(\widetilde{F}) ; \mathbb{Z})$ we may twist the spin structure $\widetilde{\mathfrak{s}}$ by the dual of this circle in $\operatorname{Hom}\left(H_{1}(W-v(\widetilde{F}) ; \mathbb{Z}), \mathbb{Z}_{2}\right)$ to obtain a spin structure that extends over $W$.

In the special case of a $2-$ fold cover where the branch set is a disk, the cohomology $H^{1}\left(W ; \mathbb{Z}_{2}\right)$ vanishes, so there is a unique spin structure on $W$. The restriction of this spin structure to $Y$ is readily characterized as the unique invariant $\operatorname{spin}^{c}$ structure on $Y$, because the conjugation of $\operatorname{spin}^{c}$ structures acts on the odd-order group $H^{2}(Y ; \mathbb{Z})$ by multiplication by -1 . This observation is very convenient, because the methods of Section 6 make it easy to identify this invariant $\operatorname{spin}^{c}$ structure. 


\section{$3 \tau$-invariants of knots in rational homology spheres}

In this section we adapt the discussion from Ozsváth-Szabó [23] to nullhomologous knots in $\mathbb{Q} \mathrm{HS}^{3}$. Our main aim is the proof of Theorem 1.1. We begin by collecting some standard Heegaard Floer homology facts. See Ozsváth-Szabó [25; 24; 26] for more details.

Let $Y$ be a $\mathbb{Q} \mathrm{HS}^{3}$ and $K \subset Y$ an oriented null-homologous knot in $Y$. We associate to the pair $(Y, K)$ a $2 n$-pointed Heegaard diagram ie a tuple $(\Sigma, \vec{\alpha}, \vec{\beta}, \vec{w}, \vec{z})$ associated to a handlebody decomposition of $Y$ coming from a generic self-indexing Morse function $f: Y \rightarrow \mathbb{R}$ with $\left|f^{-1}(0)\right|=\left|f^{-1}(3)\right|=n$. Label the index 0 critical points $a_{1}, \ldots a_{n}$ and the index 3 critical points $b_{1}, \ldots b_{n}$. Here,

- $\Sigma=f^{-1}\left(\frac{3}{2}\right)$ is the Heegaard surface,

- $\vec{\alpha}=\left(\alpha_{1}, \alpha_{2}, \ldots, \alpha_{g+n-1}\right)$ are the coattaching circles of the 1-handles,

- $\vec{\beta}=\left(\beta_{1}, \beta_{2}, \ldots, \beta_{g+n-1}\right)$ are the attaching circles of the 2-handles and

- $\vec{w}=\left(w_{1}, \ldots, w_{n}\right)$ and $\vec{z}=\left(z_{1}, \ldots, z_{n}\right)$ are two $n$-tuples of points (all distinct) on $\Sigma-\vec{\alpha}-\vec{\beta}$, where $w_{i}$ specifies a unique flowline $\gamma_{i}$ from $b_{i}$ to $a_{i}$ (the one that intersects $\Sigma$ at $w_{i}$ ) and $z_{i}$ specifies a unique flowline $\eta_{i}$ from $b_{\sigma(i)}$ to $a_{i}$ ( $\sigma$ some permutation of $\{1, \ldots, n\}$ ).

Then $K$ is uniquely determined by this data as the isotopy class of

$$
\bigcup_{i=1}^{n}-\gamma_{i} \cup \eta_{i}
$$

In the case of a 2-pointed Heegaard diagram, one produces the $\mathbb{Z} \oplus \mathbb{Z}$-filtered chain complex $\mathrm{CFK}^{\infty}(Y, K)$, a chain complex

- over $^{2} \mathbb{Z}_{2}\left[U, U^{-1}\right]$,

- whose generators are elements of the form $U^{n} \mathbf{x}$, where $\mathbf{x} \in \mathbb{T}_{\alpha} \cap \mathbb{T}_{\beta}$ is an intersection point between the $\alpha$ and $\beta$ tori in $\operatorname{Sym}^{g}(\Sigma)$, and $n \in \mathbb{Z}$,

- whose boundary map is given by

$$
\partial^{\infty}(\mathbf{x})=\sum_{\mathbf{y} \in \mathbb{T}_{\alpha} \cap \mathbb{T}_{\beta}} \sum_{\left\{\phi \in \pi_{2}(\mathbf{x}, \mathbf{y}) \mid \mu(\phi)=1\right\}} \#(\widehat{\mathcal{M}}(\phi)) U^{n_{w}(\phi)} \mathbf{y}
$$

where $\#(\widehat{\mathcal{M}}(\phi))$ is counted modulo $2 .^{3}$

${ }^{2}$ We define all Heegaard Floer chain complexes with $\mathbb{Z}_{2}$ coefficients in order to avoid orienting moduli spaces.

${ }^{3}$ Our notation matches that of [24]. 
Each intersection point $\mathbf{x}$ is assigned

- an element $\underline{\mathfrak{s}}(\mathbf{x}) \in \operatorname{Spin}^{c}\left(Y_{0}(K)\right)$ (where $Y_{0}(K)$ denotes 0 -surgery on $K$ ),

- $\mathfrak{s}(\mathbf{x}) \in \operatorname{Spin}^{c}(Y)$,

- an Alexander grading $\mathbf{A}(\mathbf{x}) \in \mathbb{Z}$ and

- a Maslov grading $\mathbf{M}(\mathbf{x}) \in \mathbb{Q}$.

Furthermore, the first three assignments are related by the natural splitting

$$
\operatorname{Spin}^{c}\left(Y_{0}\right)=\operatorname{Spin}^{c}(Y) \oplus \mathbb{Z} P D[\mu],
$$

where $\mu$ is a choice of oriented meridian for $K$. Thinking of a $\operatorname{spin}^{c}$ structure as a homology class of non-vanishing vector field (see Turaev [33], also [25, Section 2.6] and [24, Section 2.3]), the map

$$
\underline{\mathfrak{s}} \rightarrow \mathfrak{s}
$$

to the first summand is the unique extension to $Y$ of the restriction of $\underline{\mathfrak{s}}$ to the knot complement, while the map

$$
\underline{\mathfrak{s}} \rightarrow \mathbf{A}
$$

to the second is one-half of the evaluation of $c_{1}(\underline{\mathfrak{s}})$ on the surface obtained by cappingoff a Seifert surface for $K$ with the core of the 0 -surgery.

The Maslov grading is the absolute $\mathbb{Q}$ homological grading defined in Ozsváth-Szabó [27].

The $\mathbb{Z} \oplus \mathbb{Z}$-filtration on the $\mathrm{CFK}^{\infty}$ chain complex arises from an assignment of a filtration bigrading $A_{w} \times A_{z}$ to the chain complex generators (not to be confused with the $\mathbf{A} \times \mathbf{M}$ bigrading discussed in Section 6). This filtration bigrading is uniquely specified by the rules:

- $A_{w}(\mathbf{x})=0 \quad \forall \quad \mathbf{x} \in \mathbb{T}_{\alpha} \cap \mathbb{T}_{\beta}$,

- $A_{z}(\mathbf{x})=\mathbf{A}(\mathbf{x}) \quad \forall \mathbf{x} \in \mathbb{T}_{\alpha} \cap \mathbb{T}_{\beta}$,

- $A_{w}\left(U^{n} \mathbf{x}\right)=A_{w}(\mathbf{x})-n$,

- and $A_{z}\left(U^{n} \mathbf{x}\right)=A_{z}(\mathbf{x})-n$.

In addition, we remark that $\mathbf{M}\left(U^{n} \mathbf{x}\right)=\mathbf{M}(\mathbf{x})-2 n$. The $E^{2}$ term of the associated spectral sequence is $\operatorname{HFK}^{\infty}(Y, K)$, and the $E^{\infty}$ term is $\operatorname{HF}^{\infty}(Y)$.

The $\widehat{C F}(Y, K)$ chain complex is now the quotient complex corresponding to the $A_{w}=0$ slice of the $\operatorname{CFK}^{\infty}(Y, K)$ chain complex. $\widehat{C F}(Y, K)$ has a $\mathbb{Z}$-filtration coming from the Alexander grading $A_{z}=\mathbf{A}$. The associated graded chain complex of 
this filtered complex is $\widehat{C F K}(Y, K)$. Note that the $E^{2}$ term of the spectral sequence induced by the filtration on $\widehat{C F}(Y, K)$ is $\widehat{H F K}(Y, K)$, and the $E^{\infty}$ term is $\widehat{H F}(Y)$. Let $\mathcal{F}(Y, K, \mathfrak{s}, \ell)$ denote the subcomplex of $\widehat{C F}(Y, K)$ generated by elements $\mathbf{x} \in$ $\widehat{C F}(Y ; K)$ with $\mathfrak{s}(\mathbf{x})=\mathfrak{s}$ and $\mathbf{A}(\mathbf{x}) \leq \ell$. The inclusion $\mathcal{F}(Y, K, \mathfrak{s}, \ell) \rightarrow \widehat{C F}(Y, \mathfrak{s})$, induces a morphism on homology denoted by $\imath_{K, \mathfrak{s}}^{\ell}$.

Recall that all of the aforementioned chain complexes (and, hence, all terms in the associated spectral sequences) split according to $\operatorname{spin}^{c}$ structures on $Y$ :

$$
\operatorname{CFK}(Y, K)=\underset{\left\{\mathfrak{s} \in \operatorname{Spin}^{c}(Y)\right\}}{\oplus} \operatorname{CFK}(Y, K, \mathfrak{s}) .
$$

Furthermore, $\widehat{H F}(Y, \mathfrak{s})$ is isomorphic to $\mathbb{Z}_{2}$ if $Y$ is an L-space; for a general $\mathbb{Q} \mathrm{HS}^{3}$ $Y$, it contains a distinguished $\mathbb{Z}_{2}$ summand, which is in the image of $\operatorname{HF}^{\infty}(Y, \mathfrak{s})$. We denote this copy of $\mathbb{Z}_{2}$ by $\widehat{H F}_{U}(Y, \mathfrak{s})$.

Definition 3.1 The correction term for a torsion $\operatorname{spin}^{c}$ structure $\mathfrak{s}$, denoted $d_{\mathfrak{s}}(Y)$, is the absolute $\mathbb{Q}$ homological grading, $\mathbf{M}$, of $\widehat{H F}_{U}(Y, \mathfrak{s})$.

Definition 3.2 $\tau_{\mathfrak{s}}(Y, K)=\tau_{\mathfrak{s}}(K)$ is the minimal value of $\ell$ for which the image of $\imath_{K, \mathfrak{s}}^{\ell}$ has nontrivial projection to $\widehat{H F}_{U}(Y, \mathfrak{s})$.

There is an interpretation of $\tau$ in terms of surgeries on $K$. Denote by $Q(K, \mathfrak{s}, \ell)$ the quotient complex $\widehat{C F}(Y, \mathfrak{s}) / \mathcal{F}(Y, K, \mathfrak{s}, \ell)$ and by $p_{K, \mathfrak{s}}^{\ell}$ the map induced by the projection on homology. For any integer $n$ let

$$
\widehat{F}_{n, \mathfrak{s}, \ell}: \widehat{H F}(Y, \mathfrak{s}) \rightarrow \widehat{H F}\left(Y_{-n}(K), \mathfrak{s}_{\ell}\right)
$$

denote the map associated to the two-handle cobordism, where the cobordism is endowed with the $\operatorname{spin}^{c}$ structure $\mathfrak{t}_{\ell}$, whose restriction to $Y$ is $\mathfrak{s}$ and which satisfies

$$
\left\langle c_{1}\left(\mathfrak{t}_{\ell}\right),[\widehat{S}]\right\rangle-n=2 \ell ;
$$

here $\widehat{S}$ denotes the surface obtained by capping-off a Seifert surface for $K$ with the core of the two-handle. These conditions uniquely specify $\mathfrak{t}_{\ell}$ and hence also the induced $\operatorname{spin}^{c}$ structure $\mathfrak{s}_{\ell}$ on the surgery.

Proposition 3.3 If $\ell<\tau_{\mathfrak{s}}(K)$, then $\widehat{F}_{n, \mathfrak{s}, \ell}\left(\widehat{H F}_{U}(Y, \mathfrak{s})\right)$ is nontrivial for all sufficiently large $n$.

Proof Fix a 2-pointed Heegaard diagram for the pair $(Y, K)$ as well as a $\operatorname{spin}^{c}$ structure $\mathfrak{s} \in \operatorname{Spin}^{c}(Y)$. Let $C_{\mathfrak{s}}$ denote the $\mathrm{CFK}^{\infty}(Y, K, \mathfrak{s})$ chain complex. Then 
- $C_{\mathfrak{s}}\left\{A_{w}=0\right\}$ represents $\widehat{C F}(Y, \mathfrak{s})$ and

- $C_{\mathfrak{s}}\left\{A_{w}=0, A_{z} \leq \ell\right\}$ represents $\mathcal{F}(Y, K, \mathfrak{s}, \ell)$.

To relate this to the surgery recall [24, Theorem 4.1] (and the discussion following it), which says that for all sufficiently large $n, \widehat{C F}\left(Y_{-n}(K), \mathfrak{s}_{\ell}\right)$ is identified with $C_{\mathfrak{s}}\left\{\min \left(A_{w}, A_{z}-\ell\right)=0\right\}$. Furthermore, under this identification the map $\widehat{F}_{n, \mathfrak{s}, \ell}$ is induced by the projection $f: C_{\mathfrak{s}}\left\{A_{w}=0\right\} \rightarrow C_{\mathfrak{s}}\left\{\min \left(A_{w}, A_{z}-\ell\right)=0\right\}$. This yields the following commutative diagram:

$$
\begin{aligned}
& 0 \rightarrow C_{\mathfrak{s}}\left\{\begin{array}{l}
A_{w}=0 \\
A_{z} \leq \ell
\end{array}\right\} \rightarrow \quad C_{\mathfrak{s}\left\{A_{w}=0\right\}} \quad \rightarrow Q(K, \mathfrak{s}, \ell) \rightarrow 0 \\
& \downarrow \begin{array}{l}
\downarrow \\
0 \rightarrow C_{\mathfrak{s}}\left\{\begin{array}{l}
A_{w} \geq 0 \\
A_{z}=\ell
\end{array}\right\} \rightarrow C_{\mathfrak{s}}\left\{\min \left(A_{w}, A_{z}-\ell\right)=0\right\}
\end{array} \quad \rightarrow Q(K, \mathfrak{s}, \ell) \rightarrow 0
\end{aligned}
$$

Since for $\ell<\tau_{\mathfrak{s}}(K)$ the projection of the image of $\imath_{K, \mathfrak{s}}^{\ell}$ into $\widehat{H F}_{U}(Y, \mathfrak{s})$ is trivial, $p_{K, \mathfrak{s}}^{\ell}$ and therefore $\widehat{F}_{n, \mathfrak{s}, \ell}$ are nontrivial on $\widehat{H F}_{U}(Y, \mathfrak{s})$.

The following properties of $\tau$ are important for our applications.

\section{Proposition 3.4}

(1) Let $\left(Y_{i}, K_{i}\right)$ be oriented knots and $\mathfrak{s}_{i} \in \operatorname{Spin}^{c}\left(Y_{i}\right), i=1,2$. Then

$$
\tau_{\mathfrak{s}_{1} \# \mathfrak{s}_{2}}\left(Y_{1} \# Y_{2}, K_{1} \# K_{2}\right)=\tau_{\mathfrak{s}_{1}}\left(Y_{1}, K_{1}\right)+\tau_{\mathfrak{s}_{2}}\left(Y_{2}, K_{2}\right) .
$$

(2) If $(Y, K)$ is an oriented knot and $\mathfrak{s} \in \operatorname{Spin}^{c}(Y)$, then $\tau_{\mathfrak{s}}(-Y, K)=-\tau_{\mathfrak{s}}(Y, K)$.

\section{Proof}

(1) This follows from the Künneth Theorem for the knot filtration [24, Theorem 7.1] and from the fact that the tensor product of two vector space morphisms is nontrivial if and only if both of the morphisms are nontrivial.

(2) If $(\Sigma, \vec{\alpha}, \vec{\beta}, w, z)$ is a doubly pointed Heegaard diagram for $(Y, K)$, then $(-\Sigma, \vec{\alpha}, \vec{\beta}, w, z)$ is a diagram for $(-Y, K)$. If $\mathbf{x}, \mathbf{y} \in \mathbb{T}_{\alpha} \cap \mathbb{T}_{\beta}$ and $\phi \in \pi_{2}(\mathbf{x}, \mathbf{y})$, then consider the homotopy class $\phi^{\prime}$ of disks from $\mathbf{y}$ to $\mathbf{x}$, obtained from $\phi$ by precomposing with the complex conjugation. If $J_{S}$ is any one-parameter family of complex structures, then $J_{s}$-holomorphic representatives for $\phi$ are in one-to-one correspondence with $-J_{S}$-holomorphic representatives for $\phi^{\prime}$. This correspondence induces a duality map $\mathcal{D}: \widehat{C F}_{*}(Y, \mathfrak{s}) \rightarrow \widehat{C F}^{*}(-Y, \mathfrak{s})$ which 
takes elements in $A_{z}$ filtration level $\ell$ to $A_{z}$ filtration level $-\ell$; here $\widehat{C F}_{*}$ denotes the chain complex and $\widehat{C F}^{*}$ the cochain complex obtained by applying the $\operatorname{Hom}\left(\cdot, \mathbb{Z}_{2}\right)$ functor. Applying the duality isomorphism to the short exact sequence corresponding to the inclusion $\mathcal{F}_{*}(Y, K, \mathfrak{s}, \ell) \rightarrow \widehat{C F}_{*}(Y, \mathfrak{s})$ yields the following exact sequence

$0 \rightarrow Q^{*}(-Y, K, \mathfrak{s},-\ell-1) \rightarrow \widehat{C F}^{*}(-Y, \mathfrak{s}) \rightarrow \mathcal{F}^{*}(-Y, K, \mathfrak{s},-\ell-1) \rightarrow 0$.

The image of the left map in homology has trivial projection into $\widehat{H F}_{U}^{*}(-Y, \mathfrak{s})$ for all $\ell<\tau_{\mathfrak{s}}(Y, K)$, so $\mathcal{F}_{*}(-Y, K, \mathfrak{s},-\ell-1)$ maps nontrivially to $\widehat{H F}_{U, *}(-Y)$ for all $\ell$ satisfying $-\ell-1 \geq-\tau_{\mathfrak{s}}(Y, K)$. It follows that $\tau_{\mathfrak{s}}(-Y, K)=-\tau_{\mathfrak{s}}(Y, K)$.

Thus the proposition is proved.

Proposition 3.5 Let $W$ be a $\mathbb{Q} \mathrm{HB}^{4}$ with boundary $Y$. Then there exist $\mathfrak{s}_{0} \in \operatorname{Spin}^{c}(Y)$ and a subgroup $G<H^{2}(Y ; \mathbb{Z})$ with $|G|^{2}=\left|H^{2}(Y ; \mathbb{Z})\right|$ such that for any $\mathfrak{s} \in \mathfrak{s}_{0}+G$ the following hold:

(1) $d_{\mathfrak{s}}(Y)=0$ and

(2) $\mathfrak{s}$ extends to $\mathfrak{t} \in \operatorname{Spin}^{c}(W)$ and the map $\widehat{F}_{W-B^{4}, \mathfrak{\circ}}: \widehat{H F}\left(S^{3}\right) \rightarrow \widehat{H F}_{U}(Y, \mathfrak{s})$ is an isomorphism.

Moreover, if $W$ is spin, then $\mathfrak{s}_{0}$ can be chosen to be a spin structure.

Proof The first statement follows from Owens-Strle [21, Proposition 4.1]. For the second note, that

$$
F_{W-B^{4}, \mathfrak{t}}^{\infty}: \operatorname{HF}^{\infty}\left(S^{3}\right) \rightarrow \operatorname{HF}^{\infty}(Y, \mathfrak{s})
$$

is an isomorphism, according to Ozsváth-Szabó [27, Theorem 9.6]. Since $W$ is a $\mathbb{Q} \mathrm{HB}^{4}$, this map preserves absolute grading, hence the map in degree zero is an isomorphism.

Theorem 3.6 Let $W$ be a $\mathbb{Q} \mathrm{HB}^{4}$ with boundary $Y$ and $S \subset W$ a surface whose boundary is a knot $K \subset Y$. If $\mathfrak{s} \in \operatorname{Spin}^{c}(Y)$ extends over $W$ and $d_{\mathfrak{s}}(Y)=0$, then $g(S) \geq\left|\tau_{\mathfrak{s}}(K)\right|$.

Proof If $g(S)=0$ we may replace $K$ with the connected sum of $K$ and the trefoil of appropriate handedness so as to increase both sides of the claimed inequality by 1. Thus we may assume $g(S)>0$. We may further assume that $\tau_{\mathfrak{s}}(Y, K) \geq 0$ by changing the orientation of $Y$ if necessary (see Proposition 3.4). 
Choose $\ell<\tau_{\mathfrak{s}}(K)$ and let $X$ be obtained from $W$ by adding a two-handle along $K$ with framing $-n$, where $n$ is large enough so that Proposition 3.3 applies. Endow $X$ with a $\operatorname{spin}^{c}$ structure $\mathfrak{t}$, whose restriction to $Y$ is $\mathfrak{s}$ and whose restriction to the two-handle cobordism agrees with $\mathfrak{t}_{\ell}$ (defined before Proposition 3.3). Then

$$
\left\langle c_{1}(\mathfrak{t}),[\widehat{S}]\right\rangle-n=2 \ell,
$$

where $\widehat{S}$ denotes the surface obtained by capping-off $S$ with the core of the twohandle. The map $\widehat{F}_{X-B^{4}, \mathrm{t}}: \widehat{H F}\left(S^{3}\right) \rightarrow \widehat{H F}\left(Y_{-n}(K), \mathfrak{s}_{\ell}\right)$ is nontrivial, since it is the composition of the map induced by $W$ with image $\widehat{H F}_{U}(Y, \mathfrak{s})$ and the map induced by the two-handle cobordism that is nontrivial on $\widehat{H F}_{U}(Y, \mathfrak{s})$ by Proposition 3.3.

Now we split the cobordism $X$ differently. Let $N$ be a tubular neighborhood of $\widehat{S}$. Then by [23, Lemma 3.5] the map induced by the cobordism $N-B^{4}$ and $\operatorname{spin}^{c}$ structure $\mathfrak{t}$ is trivial unless

$$
\left\langle c_{1}(\mathfrak{t}),[\widehat{S}]\right\rangle-n \leq 2 g(S)-2,
$$

from which we conclude $\left|\tau_{\mathfrak{s}}(K)\right| \leq g(S)$.

We note that Theorem 1.1 is an immediate consequence of Proposition 3.5 and Theorem 3.6.

\section{Obstructions to finite concordance order}

Using Theorem 1.1 to test whether a given knot is slice might in principle require a good deal of calculation. This is because one does not know in advance the subgroup $G$ with $|G|^{2}=\left|H^{2}(Y ; \mathbb{Z})\right|$ (referenced in the theorem) on which all $\tau$ and $d$-invariants must vanish; hence, to rule out the existence of such a subgroup, one must find all subgroups $G$ of the appropriate order and verify that, indeed, either $\tau_{\mathfrak{s}}(\widetilde{K}), d_{\mathfrak{s}}(Y) \neq 0$ for some $\mathfrak{s} \in\left(\mathfrak{s}_{0}+G\right)$ in each case.

This can be computationally formidable; as an example consider the 2-bridge knot $K_{45,17}$. The $d$-invariant calculations in Jabuka-Naik [9] left open the possibility that $K=\#_{4} K_{45,17}$ might be slice, because there are a few order $45^{2}$ subgroups of $(\mathbb{Z} / 45)^{4}$ on which the $d$-invariant vanishes. Similarly, the $\tau$ obstruction for $K$ necessitated the computation of $\tau_{\mathfrak{s}}$ for $\mathfrak{s} \in \mathfrak{s}_{0}+G$ on all order $45^{2}$ subgroups $G$ of $(\mathbb{Z} / 45)^{4}$, which we did using the program pari-gp [32]. The computation took quite a while (there are $9,745,346$ such subgroups), and indeed there exist nonzero $\tau$-invariants in each one so that $K$ is not slice. The complexity of such calculations clearly gets out of hand rapidly. 
Inspired by Jabuka-Naik's idea (see [9, Obstruction 5.1]) of looking at smaller-order, more computationally-accessible, subgroups of $H^{2}(Y ; \mathbb{Z})$, we developed two simple obstructions to a knot having finite smooth concordance order. Although at first glance these obstructions seem much weaker than the original obstructions provided by Theorem 1.1, they have had remarkable success on the class of knots upon which we were able to perform calculations. In fact, our obstructions were able to show that all of the 2-bridge knots considered in [9] have infinite concordance order. We also determined the (previously unknown) smooth concordance order of all 2-bridge knots with crossing number at most 12 . The obstructions and examples follow in the next two sections. The final section is devoted to a brief explanation of how the $\tau$ calculations were performed.

We will find the following notation useful in what follows. If $f: A \rightarrow \mathbb{Q}$ is a function on a finite abelian group and $H<A$ is any subgroup we let $S_{H}(f)=\sum_{h \in H} f(h)$.

Definition 4.1 Let $K \subset S^{3}$ be a knot, $Y$ the double-branched cover of $K, \widetilde{K}$ the preimage of $K$ in $Y$, and $p \in \mathbb{Z}_{+}$either a prime or 1 . Fix an affine identification of $\operatorname{Spin}^{c}(Y)$ with $A=H^{2}(Y ; \mathbb{Z})$ such that the distinguished spin structure $\mathfrak{s}_{0}$ mentioned in Lemma 2.1 corresponds to 0.

Let $\mathcal{G}_{p}$ denote the set of all order- $p$ subgroups of $A$. Define

$$
\mathcal{T}_{p}(K):=\min \left\{\left|\sum_{H \in \mathcal{G}_{p}} n_{H} S_{H}(\tau(Y, \widetilde{K}))\right|: n_{H} \in \mathbb{Z}_{\geq 0}, \sum_{H \in \mathcal{G}_{p}} n_{H}>0\right\}
$$

if $p$ divides $\operatorname{det}(K)$ and $\mathcal{T}_{p}(K):=0$ otherwise;

$$
\mathcal{D}_{p}(K):=\min \left\{\left|\sum_{H \in \mathcal{G}_{p}} n_{H} S_{H}(d(Y))\right|: n_{H} \in \mathbb{Z}_{\geq 0}, \sum_{H \in \mathcal{G}_{p}} n_{H}>0\right\}
$$

if $p$ divides $\operatorname{det}(K)$ and $\mathcal{D}_{p}(K):=0$ otherwise.

It is worthwhile to remark at this point that the definitions of $\mathcal{T}_{p}$ and $\mathcal{D}_{p}$ are considerably simpler when $A=H^{2}(Y ; \mathbb{Z})$ is cyclic (as is the case for all 2-bridge knots). In this case, there is a unique subgroup of $A$ of order $p$ for each $p \operatorname{dividing} \operatorname{det}(K)$, and hence $\mathcal{T}_{p}$ (resp. $\mathcal{D}_{p}$ ) is just the absolute value of

$$
\sum_{\left\{\mathfrak{s} \in \operatorname{Spin}^{c}(Y) \mid \mathfrak{s} \text { has order } \mathrm{p}\right\}} \tau_{\mathfrak{s}}(\widetilde{K})\left(\text { resp. } d_{\mathfrak{s}}(Y)\right) .
$$

More generally we note that $\mathcal{T}_{p}$ or $\mathcal{D}_{p}$ is nonzero if the sums $S_{H}$ of the corresponding invariants are nonzero and of the same sign on all the subgroups $H$ of order $p$. 
Note that the invariant $\mathcal{D}_{1}$ is $\frac{1}{2} \delta$, where $\delta$ is Manolescu-Owens' concordance invariant [18].

The rest of this section will be devoted to proving Theorem 1.2, the statement of which we repeat here for convenience.

Theorem (Theorem 1.2) Let $K \subset S^{3}$ be a knot and $p \in \mathbb{Z}_{+}$prime or 1 . If there exists a positive $n \in \mathbb{Z}$ such that $\#_{n} K$ is smoothly slice, then $\mathcal{T}_{p}(K)=\mathcal{D}_{p}(K)=0$.

Proof The proof is essentially Theorem 1.1 in the case $q^{r}=2$ combined with the elementary observation that a finite abelian group of order $m$ contains a subgroup of order $p$ ( $p$ prime) whenever $p \mid m$.

Assume that $K$ has finite smooth concordance order, $n$, and fix a prime $p$.

Let $A$ denote $H^{2}(Y ; \mathbb{Z})$, where $Y$ is the double-branched cover of $K$. By Theorem 1.1 in the case $q^{r}=2$, there exists a subgroup $G<A^{n}$ with $|G|=|A|^{n / 2}$ on which $\tau$ and $d$ vanish identically (here, again, we have fixed an affine identification of $\operatorname{Spin}^{c}(Y)$ with $\left.H^{2}(Y ; \mathbb{Z})\right)$. As usual, $\widetilde{K}$ denotes the preimage of $K$ in $Y$.

We represent an element $g \in G$ by $g=\left(g_{1}, \ldots, g_{n}\right)$ where $g_{i} \in A$. Note that

$$
\tau_{g}\left(\#_{n} \widetilde{K}\right)=\sum_{i=1}^{n} \tau_{g_{i}}(\widetilde{K})
$$

and

$$
d_{g}\left(\#_{n} Y\right)=\sum_{i=1}^{n} d_{g_{i}}(Y)
$$

(see Proposition 3.4 and [9, Section 2.3]).

Consider a finite abelian group, $A$, and suppose that we have a function $f: A \rightarrow \mathbb{Q}$. We have in mind $A=H^{2}(Y ; \mathbb{Z})$ and $f$ either the $\tau$ or $d$-invariant subject to a fixed affine identification of $\operatorname{Spin}^{c}(Y)$ with $A$. Given such an identification, there is a straightforward extension that identifies $\operatorname{Spin}^{c}\left(\#_{n} Y\right)$ with $A^{n}$. For $n \in \mathbb{N}$, denote by $f^{(n)}: A^{n} \rightarrow \mathbb{Q}$ the function $f^{(n)}\left(g_{1}, \ldots, g_{n}\right)=f\left(g_{1}\right)+\cdots+f\left(g_{n}\right)$.

We assume without loss of generality that $p \operatorname{divides} \operatorname{det}(K)$ and hence $|G|$. Therefore, $G$ contains an element of order $p$, say $g=\left(g_{1}, \ldots g_{n}\right)$. Note that $\forall i \in\{1, \ldots n\} g_{i}$ has order dividing $p$ and at least one of the $g_{i}$ has order $p$. Let $\langle g\rangle=\{0 g, g, \ldots,(p-1) g\}$ denote the cyclic subgroup in $G$ generated by $g$ and $G_{i}$ denote the cyclic subgroup in $A$ generated by $g_{i}$. Theorem 1.1 tells us that $f^{(n)}$ vanishes identically on $G$, hence on $\langle g\rangle$ for $f=\tau, d$. In particular,

$$
f^{(n)}(m g)=0 \text { for } m=0, \ldots, p-1 .
$$


Note that $f^{(n)}(0 g)=n f(0)=0$ implies that $\mathcal{T}_{1}=\mathcal{D}_{1}=0$, proving the proposition for $p=1$.

We now have:

$$
\begin{array}{rlrl}
f^{(n)}(m g) & =0 \forall m=0, \ldots, p-1 & \Longrightarrow \\
\sum_{m=0}^{p-1} f^{(n)}(m g) & =0 & & \Longrightarrow \\
\sum_{m=0}^{p-1} \sum_{i=1}^{n} f\left(m g_{i}\right) & =0 & \\
\sum_{i=1}^{n} \sum_{m=0}^{p-1} f\left(m g_{i}\right) & =\sum_{i=1}^{n} S_{G_{i}}(f)=0 &
\end{array}
$$

This completes the proof.

As in Jiang's proof [10] that algebraically slice knots form an infinitely generated subgroup of the concordance group, this test can be applied, one prime at a time, to show that knots are linearly independent. See Proposition 5.1 for an application of this principle.

\subsection{Further obstructions to finite concordance order}

Even when $\mathcal{T}_{p}$ and $\mathcal{D}_{p}$ vanish, it is sometimes possible, through more careful analysis, to find an obstruction to finite concordance order. The following two propositions describe tests we developed to deal with the knots $K_{77,18}, K_{81,14}, K_{125,33}$, and $K_{209,81}$, for which $\mathcal{T}_{p}$ and $\mathcal{D}_{p}$ failed to provide an obstruction.

Proposition 4.2 Let $K \subset S^{3}$ be a knot of finite concordance order and let $p$ be a prime. Denote by $Y$ the double-branched cover of $K$ and by $\widetilde{K}$ the lift of $K$ to $Y$. Suppose that the $p$-subgroup $A_{p}$ of $A=H^{2}(Y ; \mathbb{Z})$ is isomorphic to $\mathbb{Z}_{p}$ and fix some affine identification of $\operatorname{Spin}^{c}(Y)$ with $A$ that sends the spin structure on $Y$ to 0 . Then

$$
\min \left\{f(\mathfrak{s}) ; \mathfrak{s} \in A_{p}\right\}=-\max \left\{f(\mathfrak{s}) ; \mathfrak{s} \in A_{p}\right\},
$$

where $f$ denotes either $\tau(\widetilde{K})$ or $d(Y)$.

Moreover, let $\Delta( \pm M)=\left\{d \in \mathbb{Z}_{p} ; d \neq 0, f(a)=f(a+d)= \pm M\right.$ for some $\left.a \in A_{p}\right\}$, where $M$ denotes the maximum of $f$ on $A_{p}$. Then

$$
\bigcap_{d \in \Delta(M)} d^{*} \Delta(-M) \neq \varnothing,
$$

where $d^{*}$ denotes the (multiplicative) inverse of $d$ modulo $p$. 
Proof Suppose $\#_{2 n} K$ is smoothly slice. If $G<A^{2 n}$ has order $|A|^{n}$, then $G$ contains a subgroup $G_{p}$ isomorphic to $\left(\mathbb{Z}_{p}\right)^{n}$. Note that $G_{p}$ is a subgroup of $H=\left(\mathbb{Z}_{p}\right)^{2 n}<A^{2 n}$. Let $\left\{g_{i} ; i=1, \ldots, n\right\}$ be a set of generators for $G_{p}$. By elementary operations and rearrangement of summands in $H$ we may assume that the generators are of the form $g_{i}=\left(e_{i}, h_{i}\right)$ where $e_{i} \in\left(\mathbb{Z}_{p}\right)^{n}$ has the only nonzero entry in the $i^{\text {th }}$ component equal to 1 and $h_{i}=\left(h_{i j}\right) \in\left(\mathbb{Z}_{p}\right)^{n}$.

Define $M$ (resp. $m$ ) to be the maximum (resp. minimum) of $f$ on $A_{p}$. Assume contrary to the statement of the proposition that $M+m \neq 0$. Then by replacing $f$ with $-f$ if necessary, we may assume that $M+m>0$. Let $k \in \mathbb{Z}_{p}$ be such that $f(k)=M$. Then

$$
f^{(2 n)}\left(\sum_{i=1}^{n} k g_{i}\right)=n M+\sum_{j=1}^{n} f\left(\sum_{i=1}^{n} k h_{i j}\right)>0,
$$

which contradicts Theorem 1.1.

Fix $k \in \mathbb{Z}_{p}$ with $f(k)=M>0$, choose some $d \in \Delta(M)$ and let $\ell \in\{1, \ldots, n\}$. Since

$$
\begin{aligned}
& f^{(2 n)}\left(\sum_{i=1}^{n} k g_{i}\right)=n M+\sum_{j=1}^{n} f\left(a_{j}\right)=0 \text { and } \\
& f^{(2 n)}\left(d g_{\ell}+\sum_{i=1}^{n} k g_{i}\right)=0
\end{aligned}
$$

it follows that $f\left(a_{j}\right)=-M$ for all $j$ (where $a_{j}=\sum_{i=1}^{n} k h_{i j}$ ) and $d h_{\ell j} \in \Delta(-M) \cup$ $\{0\}$ for $j=1, \ldots, n$. Since $f(0)=0$ at least one $h_{i j}$ has to be nonzero; setting $\ell$ equal to this $i$ we get $h_{\ell j} \in d^{*} \Delta(-M)$. In fact, since the above did not depend on which $d \in \Delta(M)$ we chose, we conclude that

$$
h_{\ell j} \in \bigcap_{d \in \Delta(M)} d^{*} \Delta(-M),
$$

as desired.

In another direction, we can extend the definitions of $\mathcal{T}_{p}$ and $\mathcal{D}_{p}$ to include subgroups of prime-power order. More specifically, assuming notation from Definition 4.1, suppose that $p^{k}$ divides the exponent of $A$ for some $k>1$. Then, letting $\mathcal{G}_{p^{k}}$ be the set of all cyclic subgroups of $A$ of order $p^{k}$ one may define $\mathcal{T}_{p^{k}}$ and $\mathcal{D}_{p^{k}}$ as in the case $k=1$. The conclusion of Theorem 1.2 can then be strengthened to the following proposition.

Proposition 4.3 Let $K \subset S^{3}$ be a knot and $p$ a prime. Suppose that the $p$-subgroup of $H^{2}(Y ; \mathbb{Z})$ is cyclic and let $p^{m}$ be the largest power of $p$ dividing $\left|H^{2}(Y ; \mathbb{Z})\right|$. If 
some positive multiple of $K$ is smoothly slice, then $\mathcal{T}_{p^{k}}(K)=\mathcal{D}_{p^{k}}(K)=0$ for all $k \leq\left\lfloor\frac{m+1}{2}\right\rfloor$.

Proof Suppose $A$ is a finite abelian group with cyclic $p$-subgroup and $|A|=p^{m} s$, $(p, s)=1$. If $G<A^{n}$ has order $|A|^{n / 2}$, then $p^{m n / 2}$ divides the order of $G$. It follows that either $G$ has an element of order $p^{m / 2}$ (if $m$ is even) or of order $p^{(m+1) / 2}$ (if $m$ is odd). Clearly then one of the components of this element has that order.

From here the proof proceeds inductively on $k$ where each step is essentially the same as the case $k=1$; the only difference being that for $k>1$ at the end of the proof we get a sum of sums $S_{H}$, where the order of $H$ divides $p^{k}$.

It is clear how to extend the definitions of $\mathcal{T}_{p}$ and $\mathcal{D}_{p}$ to more general $p$, however care must be taken in applying such invariants when $p$ contains distinct primes. For example, if $p$ is the product of two distinct primes $q_{1}$ and $q_{2}$ then it may happen that all subgroups of order $p$ contain only elements whose components are of orders $q_{1}$ and $q_{2}$ and the above described arguments cannot be used to show that $\mathcal{T}_{p}$ and $\mathcal{D}_{p}$ obstruct finite concordance order.

\section{Examples}

There are many knots in the knot tables whose order in the smooth concordance group is unknown $[3 ; 2]$. Combining Theorem 1.1 with

- calculations of $\tau_{\mathfrak{s}}(\widetilde{K})$ for $\mathfrak{s} \in \operatorname{Spin}^{c}(Y)$ ( $Y$ the double-branched cover of a 2-bridge knot $K$ ) (described in Section 6) and

- previously-known calculations of $d_{\mathfrak{s}}(Y)$ ( $Y$ again the double-branched cover of a 2-bridge knot $K$ ) using the inductive formula in [22, Section 4.1],

we have been able to determine all of the previously-unknown smooth concordance orders for 2-bridge knots of at most 12 crossings. Some sample results are summarized in Table 1, where we also indicate which invariants provide an obstruction to finite smooth concordance order.

The most interesting example from our point of view was $K=10_{10}=K_{45,17}$. As remarked above (Section 4.1) the $d$-invariants fail to obstruct the possibility that $K$ has order 4 . However, $\tau(\widetilde{K})$ shows $K$ has infinite concordance order, since both $\mathcal{T}_{3} \neq 0$ and $\mathcal{T}_{5} \neq 0$. Further along in the Knotinfo tables, we found the 12 -crossing 2-bridge knots $K_{81,14}$ and $K_{125,33}$, for which $\mathcal{D}_{3}$ and $\mathcal{D}_{5}$ vanish (respectively). However, $\mathcal{D}_{9}$ and $\mathcal{D}_{25}$ are non-zero, and using Proposition 4.3 we showed that these 


\begin{tabular}{||c|c|c|l||}
\hline Knot $K$ & 2-bridge notation & Order of $K$ & Test \\
\hline \hline $8_{13}$ & $29 / 11$ & $\infty$ & $\mathcal{T}_{29} \neq 0$ and $\mathcal{D}_{29} \neq 0$ \\
\hline $9_{14}$ & $37 / 14$ & $\infty$ & $\mathcal{T}_{37} \neq 0$ and $\mathcal{D}_{37} \neq 0$ \\
\hline $9_{19}$ & $41 / 16$ & $\infty$ & $\mathcal{T}_{41} \neq 0$ and $\mathcal{D}_{41} \neq 0$ \\
\hline $10_{10}$ & $45 / 17$ & $\infty$ & $\begin{array}{l}\mathcal{T}_{3} \neq 0 \text { and } \mathcal{T}_{5} \neq 0 \\
\text { but } \mathcal{D}_{3}=0 \text { and } \mathcal{D}_{5}=0\end{array}$ \\
\hline $10_{13}$ & $53 / 22$ & $\infty$ & $\mathcal{T}_{53}=0$, but $\mathcal{D}_{53} \neq 0$ \\
\hline $10_{26}$ & $61 / 17$ & $\infty$ & $\mathcal{T}_{61} \neq 0$ and $\mathcal{D}_{61} \neq 0$ \\
\hline $10_{28}$ & $53 / 19$ & $\infty$ & $\mathcal{T}_{53} \neq 0$ and $\mathcal{D}_{53} \neq 0$ \\
\hline $10_{34}$ & $37 / 13$ & $\infty$ & $\mathcal{T}_{37} \neq 0$ and $\mathcal{D}_{37} \neq 0$ \\
\hline $11_{91}$ & $129 / 50$ & $\infty$ & $\mathcal{T}_{3} \neq 0$ and $\mathcal{D}_{3} \neq 0$ \\
\hline $11_{93}$ & $93 / 41$ & $\infty$ & $\mathcal{T}_{3} \neq 0$ and $\mathcal{D}_{3} \neq 0$ \\
\hline $11_{98}$ & $77 / 18$ & $\infty$ & Proposition 4.2 for $p=7,11$ \\
\hline $11_{119}$ & $77 / 34$ & $\infty$ & $\mathcal{T}_{11} \neq 0$ and $\mathcal{D}_{11} \neq 0$ \\
\hline
\end{tabular}

Table 1

knots have infinite order. Similarly for the 12-crossing 2-bridge knot $K_{209,81}$ both $\mathcal{T}$ and $\mathcal{D}$-invariants associated to 11 and 19 are zero, however the knot has infinite order by Proposition 4.2 applied to either $d$ or $\tau$-invariants. We remark that some of the knots that we treated (for instance $K_{77,18}$ and $K_{209,81}$ can be shown to have infinite topological concordance order by using the main result of Livingston-Naik [16].

\subsection{Twist Knots}

We mention the subclass of twist knots $\left(K_{p, 2}\right)$, since these were the 2-bridge knots originally addressed by Casson and Gordon. The following result is a generalization of Jiang's theorem [10] that the set of algebraically slice twist knots $K_{p^{2}, 2}, p \geq 5$ a prime, is linearly independent in the concordance group.

Proposition 5.1 All twist knots $K_{p, 2}, p \geq 3$, have infinite order in the knot concordance group except for $p=9$ (the stevedore's knot, which is slice) and $p=5$ (the figure-8 knot, which has order 2). Moreover, twist knots in any family $K_{p_{i}, 2}$ with $p_{i} \neq 5,9$, such that for each $i$ there exists a prime dividing $p_{i}$ and not dividing $p_{j}$ for $j \neq i$ are linearly independent in the concordance group. 
Proof Recall from [22, Section 4.1] (see also Owens-Strle [21]) that the $d$-invariants of the double-branched cover of $K_{p, 2}$ are

$$
d(k)=\frac{1}{4}-\frac{k^{2}}{2 p}+\left\{\begin{aligned}
\frac{1}{4} & \text { if } \frac{p+1}{2}+k \text { is even } \\
-\frac{1}{4} & \text { if } \frac{p+1}{2}+k \text { is odd }
\end{aligned}\right.
$$

for $|k| \leq \frac{p-1}{2}$, where $k=0$ corresponds to the spin structure. Then $d(0) \neq 0$ iff $p \equiv 3(\bmod 4)$. Assume now that $p \equiv 1(\bmod 4)$, let $q$ be a prime dividing $p$ and write $p=q s$. Then we have (up to sign)

$$
\mathcal{D}_{q}=2 \sum_{k=1}^{(q-1) / 2} d(k s)=\frac{q-1}{4}\left(1-\frac{s(q+1)}{6}\right)+\left\{\begin{array}{ll}
\frac{1}{2} & \text { if } \frac{q-1}{2} \text { is odd } \\
0 & \text { if } \frac{q-1}{2} \text { is even }
\end{array} .\right.
$$

Now $\mathcal{D}_{3}=0$ iff $s=3$ which corresponds to the stevedore's knot, and $\mathcal{D}_{5}=0$ iff $s=1$ which corresponds to the figure- 8 knot. Note that $\mathcal{D}_{3}<0$ if $s>3$ and $\mathcal{D}_{5}<0$ if $s>1$. Finally $\mathcal{D}_{q}<0$ for $q \geq 7$ except for $q=7$ and $s=1$, which corresponds to $p=7$ (which is not congruent to 1 modulo 4 ).

To show linear independence, suppose a knot $K=\#_{i=1}^{n} m_{i} K_{p_{i}, 2}$ is slice. Let $q_{i}$ be a prime dividing $p_{i}$ and not dividing any other $p_{j}$. If $q_{i}>7$, then $\mathcal{D}_{q_{i}}\left(K_{p_{i}, 2}\right) \neq 0$. Furthermore, since $q_{i} \nmid p_{j}$ for $i \neq j, H_{1}(Y ; \mathbb{Z})$ ( $Y$ the double-branched cover of $K$ ) has a unique subgroup of order $q_{i}$, which allows us to conclude that $\mathcal{D}_{q_{i}}(K)=$ $\mathcal{D}_{q_{i}}\left(K_{p_{i}, 2}\right) \neq 0$, contradicting the assumption that $K$ is slice. Therefore, $m_{i}=0$. If $q_{i}=3$ and $p_{i}>9$, or $q_{i}=5$ and $p_{i}>5$, or $q_{i}=7$ and $p_{i}>7$ the same conclusion holds. If $p_{i}=3$ (resp., $p_{i}=7$ ) then a direct computation shows that the $\mathcal{D}_{3}$ (resp., $D_{7}$ ) invariant is nonzero, leading to the same conclusion.

\section{Computing $\tau$-invariants for preimages of 2-bridge knots}

To compute the $\tau$-invariants associated to the preimage, $\widetilde{K}_{p, q}$, of the 2 -bridge knot $K_{p, q} \subset S^{3}$ inside its double-branched cover $-L(p, q)$, we used a computer program written in Mathematica. This program implemented the combinatorial description of the knot Floer homology of the preimage of a 2-bridge knot inside its double-branched cover presented in Grigsby [7]. We summarize the results of that paper and add some minor improvements to the combinatorial description of the $(\mathbf{A}, \mathbf{M})$ bigrading using results from the more recent work in Manolescu et al [20].

Recall that we can associate to $\widetilde{K}_{p, q} \subset-L(p, q)$ a compatible 4-pointed, genus 1 Heegaard diagram which is a twisted toroidal grid diagram consisting of two parallel curves of slope 0 and two of slope $\frac{p}{q}$, partitioning the torus into $2 p q$ cells. 
More specifically, we identify the universal cover of the torus with the plane:

$$
T^{2}:=\mathbb{R}^{2} / \mathbb{Z}^{2}
$$

The two curves of slope 0 on $T^{2}$ are the image in $T^{2}$ of the lines $y=0$ and $y=\frac{1}{2}$ and the two curves of slope $\frac{p}{q}$ are the image in $T^{2}$ of the lines $y=\frac{p}{q} x$ and $y=\frac{p}{q}\left(x-\frac{1}{2}\right)$. We now identify the toroidal grid diagram with the fundamental domain $[0,1] \times[0,1] \subset \mathbb{R}^{2},{ }^{4}$ and position our four basepoints at

$$
(\epsilon, 1-\epsilon),\left(\frac{1}{2}+\epsilon, 1-\epsilon\right),\left(\epsilon, \frac{1}{2}-\epsilon\right),\left(\frac{1}{2}+\epsilon, \frac{1}{2}-\epsilon\right)
$$

where $0<\epsilon<\min \left(\frac{1}{p}, \frac{1}{q}\right)$. See Figure 1 for the example of $K_{7,3}$.

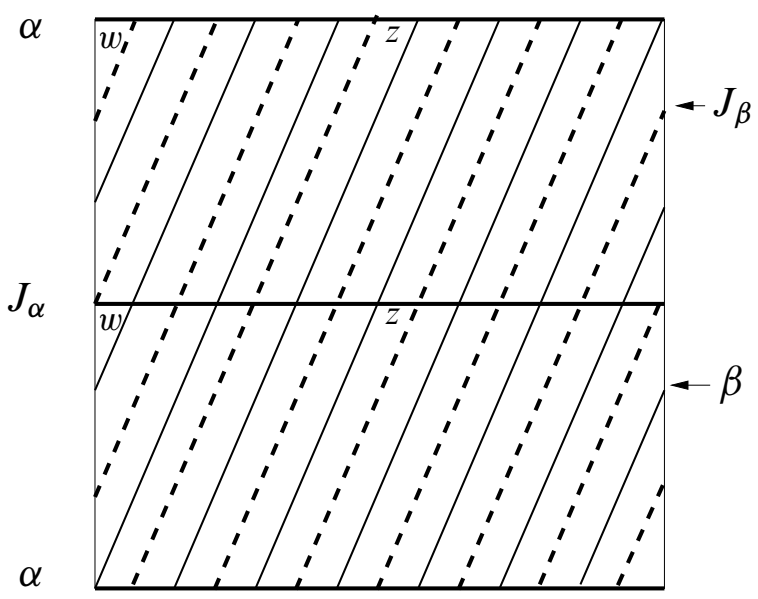

Figure 1: The twisted toroidal grid diagram which is a 4-pointed genus 1 Heegaard diagram for $\widetilde{K}_{7,3} \subset-L(7,3)$ (identify top-bottom, left-right, in the standard way).

We calculate $\tau_{\mathfrak{s}}$ for all $\mathfrak{s} \in \operatorname{Spin}^{c}(-L(p, q))$ by considering the chain complex

- whose generators are indexed by bijections between the set of slope 0 curves and the set of slope $\frac{p}{q}$ curves,

- whose differentials are given by counting parallelograms missing all $w$ basepoints and

- with a filtration induced by the Alexander grading.

${ }^{4}$ Note that by "the image in $T^{2}$ " we mean the image of these lines in the quotient $\mathbb{R}^{2} / \mathbb{Z}^{2}$ and not the intersection of these lines with the chosen fundamental domain. 
By [20, Lemma 2.1], this chain complex has the filtered chain homotopy type of $\widehat{C F}(-L(p, q)) \otimes V$ where $V$ is the chain complex with two generators, one in bigrading $(\mathbf{A}, \mathbf{M})=(0,0)$ and one in bigrading $(-1,-1)$, and no differentials. In other words,

- The associated graded complex of this chain complex is

$$
\widehat{H F K}\left(-L(p, q), \widetilde{K}_{p, q}\right) \otimes V,
$$

and

- the $E^{\infty}$ term of the spectral sequence associated to this filtered complex is

$$
\widehat{H F}(-L(p, q)) \otimes V,
$$

ie, for all $\mathfrak{s} \in \operatorname{Spin}^{c}(-L(p, q))$, there are two generators, one in bigrading $\left(\tau_{\mathfrak{s}}, d_{\mathfrak{s}}\right)$ and the other in bigrading $\left(\tau_{\mathfrak{s}}-1, d_{\mathfrak{s}}-1\right)$.

\subsection{Enumerating Generators and Differentials}

We label the intersection points as described in [7]. Namely, intersection points with $\beta$ and $J(\beta)$ along the curve $\alpha$ are cyclically labeled

$$
x_{0}, x_{0}^{\prime}, x_{1}, x_{1}^{\prime}, \ldots, x_{p-1}, x_{p-1}^{\prime},
$$

and intersection points with $\beta$ and $J(\beta)$ along the curve $J(\alpha)$ are labeled

$$
y_{0}^{\prime}, y_{0}, y_{1}^{\prime}, y_{1}, \ldots, y_{p-1}^{\prime}, y_{p-1} \text {. }
$$

See Figure 2.

The generators of the chain complex are therefore pairs of the form $\left(x_{i}, y_{j}\right)$ and $\left(x_{i}^{\prime}, y_{j}^{\prime}\right)$ for $i, j \in \mathbb{Z}_{p}$. This labeling convention is chosen so that the sum of indices constituting a generator specifies the $\operatorname{spin}^{c}$ structure in which it lives. More precisely,

$$
\left.\left(x_{i}, y_{j}\right),\left(x_{i}^{\prime}, y_{j}^{\prime}\right) \in \mathfrak{s}_{(i+j} \bmod p\right),
$$

where the subscript on $\mathfrak{s}$ denotes a particular affine identification of $\operatorname{Spin}^{c}(-L(p, q))$ with $H^{2}(-L(p, q), \mathbb{Z}) \cong \mathbb{Z}_{p}$, subject to the condition that the unique spin structure is identified with $0 \in \mathbb{Z}_{p}$.

There are $2 p^{2}$ generators, $2 p$ in each $\operatorname{spin}^{c}$ structure for $-L(p, q)$. See Table 2 to see how the generators in Figure 2 break up into $\operatorname{spin}^{c}$ structures.

After enumerating the generators, we turn our attention to enumerating the differentials. In other words, we wish to determine how many differentials (parallelograms) missing all $w$ basepoints connect $\mathbf{g}_{\mathbf{1}}$ to $\mathbf{g}_{\mathbf{2}}$, assuming that $\mathbf{g}_{\mathbf{1}}$ and $\mathbf{g}_{\mathbf{2}}$ are two generators of our chain complex 


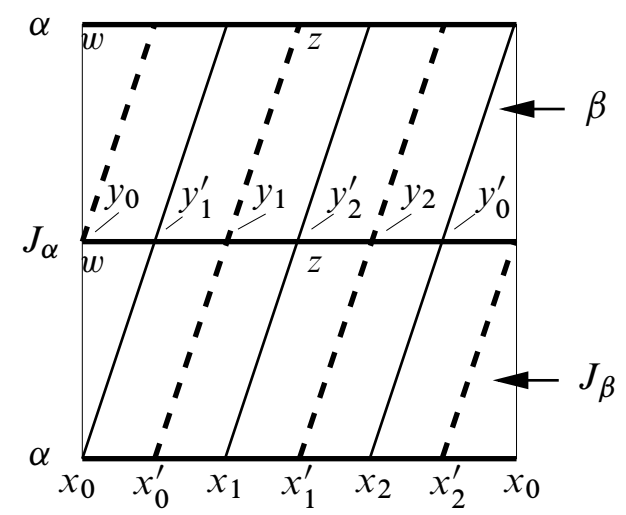

Figure 2: Labeling intersection points on the twisted toroidal grid diagram for $\widetilde{K}_{3,1} \subset-L(3,1)$.

\begin{tabular}{|c|c|c|}
\hline $\mathfrak{s}_{0}$ & $\mathfrak{s}_{1}$ & $\mathfrak{s}_{2}$ \\
\hline$\left(x_{0}, y_{0}\right)$ & $\left(x_{0}, y_{1}\right)$ & $\left(x_{0}, y_{2}\right)$ \\
$\left(x_{0}^{\prime}, y_{0}^{\prime}\right)$ & $\left(x_{0}^{\prime}, y_{1}^{\prime}\right)$ & $\left(x_{0}^{\prime}, y_{2}^{\prime}\right)$ \\
$\left(x_{1}, y_{2}\right)$ & $\left(x_{1}, y_{0}\right)$ & $\left(x_{1}, y_{1}\right)$ \\
$\left(x_{1}^{\prime}, y_{2}^{\prime}\right)$ & $\left(x_{1}^{\prime}, y_{0}^{\prime}\right)$ & $\left(x_{1}^{\prime}, y_{1}^{\prime}\right)$ \\
$\left(x_{2}, y_{1}\right)$ & $\left(x_{2}, y_{2}\right)$ & $\left(x_{2}, y_{0}\right)$ \\
$\left(x_{2}^{\prime}, y_{1}^{\prime}\right)$ & $\left(x_{2}^{\prime}, y_{2}^{\prime}\right)$ & $\left(x_{2}^{\prime}, y_{0}^{\prime}\right)$ \\
\hline
\end{tabular}

Table 2: Splitting of generators in Figure 2 according to $\operatorname{spin}^{c}$ structures

- living in the same $\operatorname{spin}^{c}$ structure and

- having relative homological (Maslov) grading ${ }^{5}$ difference 1 , ie,

$$
\mathbf{M}\left(\mathbf{g}_{1}\right)-\mathbf{M}\left(\mathbf{g}_{2}\right)=1 .
$$

Since there are always exactly 2 parallelograms connecting any two generators in the same $\operatorname{spin}^{c}$ structure with relative Maslov grading 1 (see Figure 3 and Figure 4), the only question is how many of these parallelograms (mod 2) miss all $w$ basepoints. Therefore, the multiplicity of $\mathbf{g}_{2}$ in the boundary of $\mathbf{g}_{1}$ is the reduction mod 2 of the number $(0,1$, or 2$)$ of such parallelograms containing no $w$ basepoints.

${ }^{5}$ The computation of absolute $\mathbb{Q}$ homological gradings is addressed in the next subsection. 

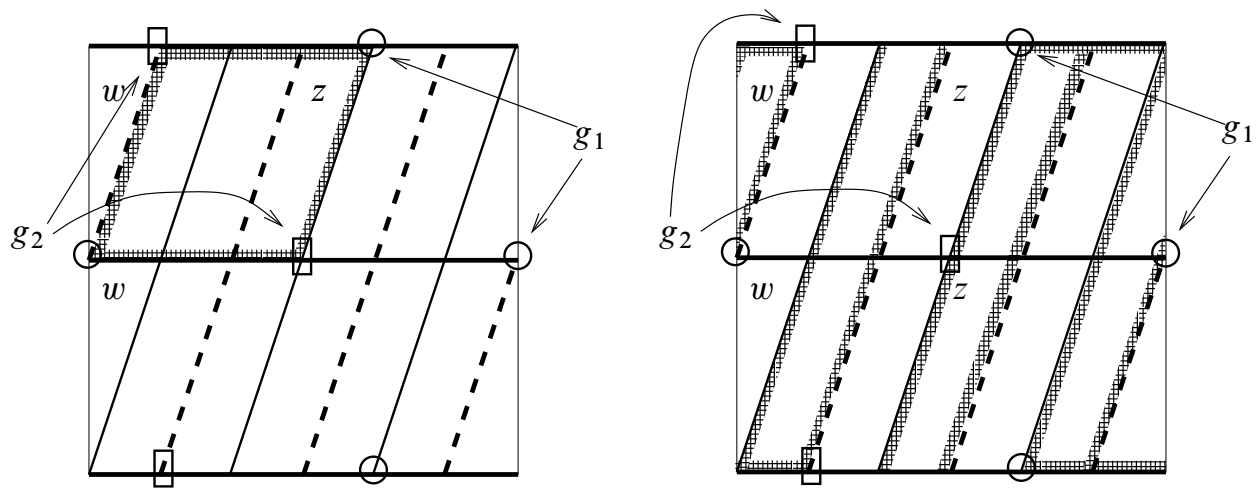

Figure 3: The two candidate differentials connecting $\mathbf{g}_{1}$ and $\mathbf{g}_{2}$ are shown. Since the second parallelogram is non-imbedded and wraps around the torus several times, we have drawn its outline only. Note that the first parallelogram misses all $w$ basepoints but the second does not. Therefore, the mod 2 multiplicity of $\mathbf{g}_{2}$ in the boundary of $\mathbf{g}_{1}$ is 1 .
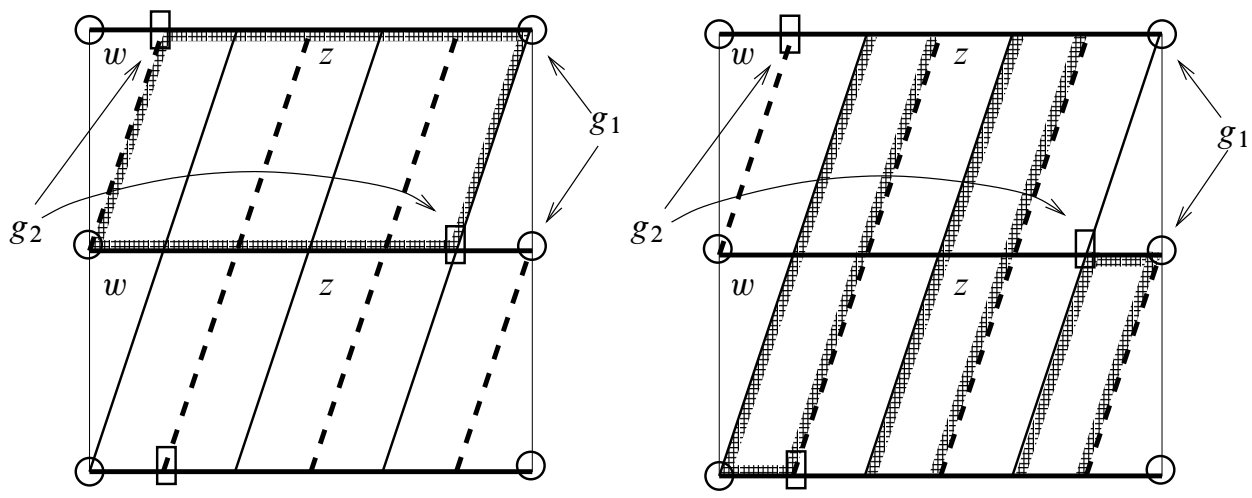

Figure 4: Here, both candidate parallelograms miss all $w$ basepoints. Therefore, the mod 2 multiplicity of $\mathbf{g}_{2}$ in the boundary of $\mathbf{g}_{\mathbf{1}}$ is 0 .

\subsection{Computing Gradings}

Denoting the set of generators of the chain complex by $\mathcal{G}$, the Alexander (filtration) grading is an assignment

$$
\mathbf{A}: \mathcal{G} \rightarrow \mathbb{Z},
$$

and the Maslov (homological) grading is an assignment

$$
\mathbf{M}: \mathcal{G} \rightarrow \mathbb{Q} \text {. }
$$


We begin by calculating the relative Maslov $\mathbb{Q}$-gradings of all generators. We do this by lifting the pair

$$
\left(-L(p, q), \widetilde{K}_{p, q}\right)
$$

to its universal cover,

$$
\left(S^{3}, \widetilde{\widetilde{K}}_{p, q}\right) \text {, }
$$

calculating the relative Maslov gradings there using the easy formula proved by Manolescu et al [20], then use Lee and Lipshitz's result in [12] relating relative Maslov gradings under covers. To nail down the absolute $\mathbb{Q}$-grading, we use the inductive formula for the correction terms in [22] to pin down the absolute grading for one generator, thus pinning down the absolute grading for all generators.

The first step in this process is understanding how to construct a Heegaard diagram for the pair

$$
\left(S^{3}, \widetilde{K}_{p, q}\right)
$$

from the Heegaard diagram for the pair

$$
\left(-L(p, q), \widetilde{K}_{p, q}\right) \text {. }
$$

The following lemma describes how to do this for any $2 n$-pointed, grid number $n$, twisted toroidal grid diagram for a knot in a lens space (not just the grid number 2 knots of interest here).

Lemma 6.1 Let $T$ be a twisted toroidal grid diagram for $K$ in $L(p, q)$. Form the universal cover, $\mathbb{R}^{2}$ of $T$, identifying $T$ with

$$
[0,1] \times[0,1] \subset \mathbb{R}^{2},
$$

the fundamental domain of the covering space action. Let $Z$ be the lattice generated by the vectors $(1,0)$ and $(0, p)$. Then

$$
\widetilde{T}=\mathbb{R}^{2} / Z
$$

is a Heegaard diagram compatible with $\widetilde{K} \subset S^{3}$, where $\widetilde{K}$ is the preimage of $K$ under the covering space projection $\pi: S^{3} \rightarrow L(p, q)$.

Proof of Lemma 6.1 The original twisted toroidal grid diagram $T$ compatible with $K \subset L(p, q)$ corresponds to a handlebody decomposition of $L(p, q)$ with one solid handlebody, $Y_{0,1}$ formed by the union of $n 0$ - and 1-handles and the other solid handlebody $Y_{2,3}$ similarly formed by the union of $n 2$-and 3 -handles. $\partial\left(Y_{0,1}\right)=$ $\partial\left(-Y_{2,3}\right)=T$. 
We will construct a Heegaard diagram for $\widetilde{K} \subset S^{3}$ by constructing a handlebody decomposition of the universal cover, $S^{3}$, compatible with this handlebody decomposition of $L(p, q)$ and the covering space action. Namely,for every $h$ in the handlebody decomposition of $L(p, q), \pi^{-1}(h)=\left\{\widetilde{h}, a \widetilde{h}, a^{2} \widetilde{h}, \ldots, a^{p-1} \widetilde{h}\right\}$ is a collection of handles in the handlebody decomposition for $S^{3}$, where $a$ is a generator of $\pi_{1}(L(p, q)) \cong \mathbb{Z}_{p}$, and

$$
\pi: S^{3} \rightarrow L(p, q)
$$

is the covering space map. The attaching maps for the lifts of the handles are uniquely specified by the condition that they commute with the covering space projection.

Applying this procedure to the handlebody decomposition associated to $T$ corresponds to cutting $Y_{0,1}$ open along some meridian and gluing $p$ copies of the resulting $D^{2} \times I$ together. From the point of view of the boundary, $T$, this corresponds to stacking $p$ copies of $T$ on top of each other (when $T$ is identified with the fundamental domain $[0,1] \times[0,1]$ in $\left.\mathbb{R}^{2}\right)$. This is precisely a description of $\widetilde{T}=\mathbb{R}^{2} / Z$.

Note that $\widetilde{T}$ is just a standard (untwisted) toroidal grid diagram for the link $\widetilde{K}$ in $S^{3}$ (in the sense of Manolescu-Ozsváth-Sarkar [19]). With this in mind, it will be convenient for us to choose a slanted fundamental domain for $\widetilde{T} \subset \mathbb{R}^{2}$, whose top and bottom edges are the same $\alpha$ curve and whose left and right edges are the same $\beta$ curve. See Figure 5.

We now recall the following fact, which is essentially Lee-Lipshitz's [12, Theorem 4.1]. See Ozsváth-Szabó [27] for a definition of $\widetilde{g r}$, the absolute $\mathbb{Q}$ homological grading.

Theorem 6.2 (Ozsváth-Szabó [12]) Let $\mathbf{g}_{1}$ and $\mathbf{g}_{2}$ be generators in torsion $\operatorname{spin}^{c}$ structures in a Heegaard Floer chain complex, $\widehat{C F}(Y)$, associated to a particular Heegaard decomposition $h d(Y)$ for $Y$.

Let $\pi: \widetilde{Y} \rightarrow Y$ be a degree $n$ covering map and $\widetilde{h d}(\widetilde{Y})$ the associated Heegaard decomposition of $\widetilde{Y}$ compatible with $\pi$.

Let $\widetilde{\mathbf{g}}_{1}$ and $\widetilde{\mathbf{g}}_{2}$ be the unique generators in $\widehat{C F}(\widetilde{Y})$ with the property that $\pi\left(\widetilde{\mathbf{g}}_{i}\right)=\mathbf{g}_{i}$ for $i=1,2$. Then

$$
\widetilde{g r}\left(\mathbf{g}_{1}\right)-\widetilde{g r}\left(\mathbf{g}_{2}\right)=\frac{1}{n}\left[\widetilde{g r}\left(\widetilde{\mathbf{g}}_{1}\right)-\widetilde{g r}\left(\widetilde{\mathbf{g}}_{2}\right)\right]
$$

This theorem allows us to compute the relative $\mathbb{Q}$ gradings between generators in the twisted toroidal grid diagram for $\left(-L(p, q), \widetilde{K}_{p, q}\right)$ by lifting the generators to the $\pi$-compatible toroidal grid diagram for $\left(S^{3}, \widetilde{\widetilde{K}}_{p, q}\right)$ and computing their relative gradings there. 

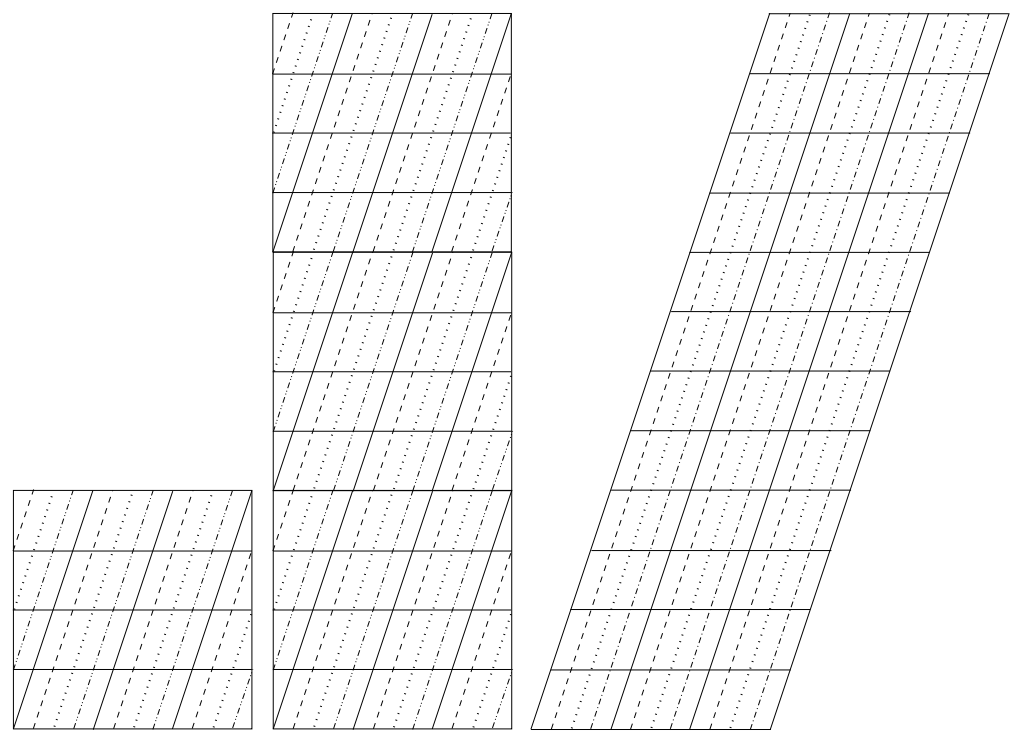

Figure 5: Constructing $\widetilde{T}$ from $T$ and adjusting the fundamental domain to identify $\widetilde{T}$ with a standard toroidal grid diagram for $\widetilde{K} \subset S^{3}$

Furthermore, in [20], an easy formula is given for determining the absolute $\mathbb{Q}$ grading for generators on a toroidal grid diagram for a link $K$ in $S^{3}$. Namely, they define a function

$$
\mathcal{I}: \mathbf{S} \times \mathbf{S} \rightarrow \mathbb{Z}_{+},
$$

where $\mathbf{S}$ is the set of finite sets of points on $\mathbb{R}^{2}:$ If $A, B \in \mathbf{S}$, then $\mathcal{I}(A, B)$ is the number of pairs $\left(a_{1}, a_{2}\right),\left(b_{1}, b_{2}\right)$ for which $\left(a_{1}, a_{2}\right) \in A,\left(b_{1}, b_{2}\right) \in B, a_{1}<a_{2}$ and $b_{1}<b_{2}$.

Upon identification of the toroidal grid diagram with a fundamental domain in $\mathbb{R}^{2}$ with the property that the left edge is one of the $\beta$ curves and the bottom edge is one of the $\alpha$ curves, they then define a function:

$$
\mathbf{M}(\mathbf{x})=\mathcal{I}(\mathbf{x}, \mathbf{x})+\mathcal{I}(\mathbb{O}, \mathbb{O})-\mathcal{I}(\mathbf{x}, \mathbb{O})-\mathcal{I}(\mathbb{O}, \mathbf{x})+1
$$

where $\mathbf{x}$ is a generator of the chain complex, and $\mathbb{O}$ is the set of $w$ basepoints. They then go on to prove that $\mathbf{M}$ (independent of the choice of identification of $\mathcal{T}$ with a fundamental domain on $\mathbb{R}^{2}$ ) is precisely $\widetilde{g r}$, the absolute homological grading, on $S^{3}$.

[20] also describes how to obtain the Alexander grading for a generator by comparing its Maslov grading with respect to the $w$ basepoints with the Maslov grading with 
respect to the $z$ basepoints:

$$
\mathbf{A}(\mathbf{x})=\frac{1}{2}\left(\mathbf{M}_{w}(\mathbf{x})-\mathbf{M}_{z}(\mathbf{x})\right)-\frac{n-1}{2},
$$

where $n$ is the grid number of $K$. Although the formula is stated in [20] only for a knot in $S^{3}$, it holds equally well for generators in a chain complex arising from a general balanced $2 n$-pointed Heegaard decomposition of a $\mathbb{Q H S}{ }^{3}$. In fact, it is a direct consequence of one of the symmetries (see $[24 ; 26]$ ) enjoyed by such a chain complex.

Namely, suppose $Y$ is a $\mathbb{Q H S}{ }^{3}$, and $K \subset Y$ is a nullhomogous knot. Fix a balanced, $2 n$-pointed Heegaard diagram for $Y$ compatible with $K$. Switching the roles of the $w$ and $z$ basepoints on the same Heegaard diagram corresponds to reversing the orientation on $K$. Furthermore, doing so induces a linear map on the chain complex sending a generator in bigrading $(i, d)$ to one in bigrading $(-i-(n-1), d-2 i-(n-1))$.

Hence, we need only determine the absolute Maslov gradings with respect to the $w$ basepoints and then again with respect to the $z$ basepoints in order to compute all of the Alexander gradings.

\section{References}

[1] A J Casson, C M Gordon, Cobordism of classical knots, from: "À la recherche de la topologie perdue”, Progr. Math. 62, Birkhäuser, Boston (1986) 181-199 MR900252

[2] J C Cha, C Livingston, Unknown values in the table of knots (2005) arXiv: math/0503125

[3] J C Cha, C Livingston, Knotinfo table of knot invariants (2006) Available at http:// www. indiana. edu/ knotinfo/

[4] T D Cochran, K E Orr, P Teichner, Knot concordance, Whitney towers and $L^{2}-$ signatures, Ann. of Math. (2) 157 (2003) 433-519 MR1973052

[5] T D Cochran, K E Orr, P Teichner, Structure in the classical knot concordance group, Comment. Math. Helv. 79 (2004) 105-123 MR2031301

[6] S K Donaldson, An application of gauge theory to four-dimensional topology, J. Differential Geom. 18 (1983) 279-315 MR710056

[7] J E Grigsby, Combinatorial description of knot Floer homology of cyclic branched covers (2006) arXiv:math/0610238

[8] J E Grigsby, Knot Floer homology in cyclic branched covers, Algebr. Geom. Topol. 6 (2006) 1355-1398 MR2253451

[9] S Jabuka, S Naik, Order in the concordance group and Heegaard Floer homology, Geom. Topol. 11 (2007) 979-994 MR2326940 
[10] B J Jiang, A simple proof that the concordance group of algebraically slice knots is infinitely generated, Proc. Amer. Math. Soc. 83 (1981) 189-192 MR620010

[11] R C Kirby, Problems in low-dimensional topology, from: "Geometric topology (Athens, GA, 1993)”, AMS/IP Stud. Adv. Math. 2, Amer. Math. Soc., Providence, RI (1997) 35-473 MR1470751

[12] D A Lee, R Lipshitz, Covering spaces and $\mathbb{Q}$ gradings on Heegaard Floer homology (2006) arXiv:math/0608001

[13] A Levine, On knots with infinite smooth concordance order (2008) arXiv: 0805.2410

[14] P Lisca, Lens spaces, rational balls and the ribbon conjecture, Geom. Topol. 11 (2007) 429-472 MR2302495

[15] P Lisca, Sums of lens spaces bounding rational balls, Algebr. Geom. Topol. 7 (2007) 2141-2164 MR2366190

[16] C Livingston, S Naik, Obstructing four-torsion in the classical knot concordance group, J. Differential Geom. 51 (1999) 1-12 MR1703602

[17] C Livingston, S Naik, Knot concordance and torsion, Asian J. Math. 5 (2001) 161-167 MR1868169

[18] C Manolescu, B Owens, A concordance invariant from the Floer homology of double branched covers, Int. Math. Res. Not. IMRN (2007) Art. ID rnm077, 21 MR2363303

[19] C Manolescu, P Ozsváth, S Sarkar, A Combinatorial Description of Knot Floer Homology (2006) arXiv:0607691

[20] C Manolescu, P Ozsváth, Z Szabó, D Thurston, On combinatorial link Floer homology, Geom. Topol. 11 (2007) 2339-2412 MR2372850

[21] B Owens, S Strle, Rational homology spheres and the four-ball genus of knots, Adv. Math. 200 (2006) 196-216 MR2199633

[22] P Ozsváth, Z Szabó, Absolutely graded Floer homologies and intersection forms for four-manifolds with boundary, Adv. Math. 173 (2003) 179-261 MR1957829

[23] P Ozsváth, Z Szabó, Knot Floer homology and the four-ball genus, Geom. Topol. 7 (2003) 615-639 MR2026543

[24] P Ozsváth, Z Szabó, Holomorphic disks and knot invariants, Adv. Math. 186 (2004) 58-116 MR2065507

[25] P Ozsváth, Z Szabó, Holomorphic disks and topological invariants for closed threemanifolds, Ann. of Math. (2) 159 (2004) 1027-1158 MR2113019

[26] P Ozsváth, Z Szabó, Holomorphic disks, link invariants and the multi-variable Alexander polynomial (2005) arXiv:math/0512286

[27] P Ozsváth, Z Szabó, Holomorphic triangles and invariants for smooth four-manifolds, Adv. Math. 202 (2006) 326-400 MR2222356 
[28] J Rasmussen, Floer homology and knot complements, $\mathrm{PhD}$ thesis, Harvard University (2003) arXiv:math/0306378

[29] J Rasmussen, Khovanov homology and the slice genus, to appear in Invent. Math. (2004) arXiv:math/0402131v1

[30] J Rasmussen, Knot polynomials and knot homologies, from: "Geometry and topology of manifolds", Fields Inst. Commun. 47, Amer. Math. Soc., Providence, RI (2005) 261-280 MR2189938

[31] S Sarkar, J Wang, A Combinatorial Description of Some Heegaard Floer Homologies (2006) arXiv:math/0607777

[32] The PARI Group, Bordeaux, PARI/GP, version 2.3.1 (2005) Available at http:// pari.math.u-bordeaux.fr/

[33] V Turaev, Torsion invariants of $\operatorname{Spin}^{c}$-structures on 3-manifolds, Math. Res. Lett. 4 (1997) 679-695 MR1484699

Department of Mathematics, Columbia University

2990 Broadway MC4406, New York, NY 10027, USA

Department of Mathematics, MS 050

Brandeis University, Waltham, MA 02454, USA

Faculty of Mathematics and Physics, University of Ljubljana

Jadranska 21, 1000 Ljubljana, Slovenia

egrigsby@math.columbia.edu, ruberman@brandeis.edu, saso.strle@fmf.uni-lj.si

Proposed: Tomasz Mrowka

Seconded: Ronald Stern, Peter Ozsváth
Received: 1 February 2007 Revised: 24 June 2008 